\title{
Evaluation of convalescent plasma versus standard of care for the treatment of COVID-19 in hospitalized patients: study protocol for a phase 2 randomized, open- label, controlled, multicenter trial
}

Elena Diago-Sempere ${ }^{1}$, José Luis Bueno², Aránzazu Sancho-López ${ }^{1 *}$, Elena Múñez Rubio ${ }^{3}$, Ferrán Torres ${ }^{4}$, Rosa Malo de Molina ${ }^{5}$, Ana Fernández-Cruz ${ }^{3}$, Isabel Salcedo de Diego ${ }^{1}$, Ana Velasco-Iglesias ${ }^{6}$, Concepción Payares-Herrera', Inmaculada Casas Flecha ${ }^{7}$, Cristina Avendaño-Solà', Rafael Duarte Palomino ${ }^{8}$, Antonio Ramos-Martínez ${ }^{3}$ and Belén Ruiz-Antorán ${ }^{1}$

\begin{abstract}
Background: COVID-19 is a respiratory disease caused by a novel coronavirus (SARS-CoV-2) and causes substantial morbidity and mortality. At the time this clinical trial was planned, there were no available vaccine or therapeutic agents with proven efficacy, but the severity of the condition prompted the use of several pharmacological and non-pharmacological interventions.

It has long been hypothesized that the use of convalescent plasma (CP) from infected patients who have developed an effective immune response is likely to be an option for the treatment of patients with a variety of severe acute respiratory infections (SARI) of viral etiology. The aim of this study is to assess the efficacy and safety of convalescent plasma in adult patients with severe COVID-19 pneumonia.

Methods/design: The ConPlas-19 study is a multicenter, randomized, open-label controlled trial. The study has been planned to include 278 adult patients hospitalized with severe COVID-19 infection not requiring mechanical ventilation (invasive or non-invasive). Subjects are randomly assigned in a 1:1 ratio (139 per treatment arm), stratified by center, to receive intravenously administered CP (single infusion) plus SOC or SOC alone, and are to be followed for 30 days. The primary endpoint of the study is the proportion of patients that progress to category 5,6 , or 7 (on the 7-point ordinal scale proposed by the WHO) at day 15. Interim analyses for efficacy and/or futility will be conducted once $20 \%, 40 \%$, and $60 \%$ of the planned sample size are enrolled and complete D15 assessment.

\footnotetext{
* Correspondence: Aranzazu.sancho@salud.madrid.org

'Clinical Pharmacology Department, Hospital Universitario Puerta de Hierro Majadahonda, Instituto de Investigación Sanitaria Puerta de Hierro - Segovia de Arana, c/ Manuel de Falla 1, 28222 Madrid, Spain

Full list of author information is available at the end of the article
}

(c) The Author(s). 2021 Open Access This article is licensed under a Creative Commons Attribution 4.0 International License, which permits use, sharing, adaptation, distribution and reproduction in any medium or format, as long as you give appropriate credit to the original author(s) and the source, provide a link to the Creative Commons licence, and indicate if changes were made. The images or other third party material in this article are included in the article's Creative Commons licence, unless indicated otherwise in a credit line to the material. If material is not included in the article's Creative Commons licence and your intended use is not permitted by statutory regulation or exceeds the permitted use, you will need to obtain permission directly from the copyright holder. To view a copy of this licence, visit http://creativecommons.org/licenses/by/4.0/ The Creative Commons Public Domain Dedication waiver (http://creativecommons.org/publicdomain/zero/1.0/) applies to the data made available in this article, unless otherwise stated in a credit line to the data. 
(Continued from previous page)

Discussion: This clinical trial is designed to evaluate the efficacy and safety of passive immunotherapy with convalescent plasma for the treatment of adult patients hospitalized with COVID-19. The results of this study are expected to contribute to establishing the potential place of CP in the therapeutics for a new viral disease.

Trial registration: ClinicalTrials.gov NCT04345523. Registered on 30 March, 2020. First posted date: April 14, 2020.

Keywords: COVID-19, Randomized, Controlled trial, Protocol, Convalescent plasma (CP), Antibodies, Neutralizing antibodies, Hospitalized patients

\section{Background}

COVID-19 is a respiratory disease caused by a novel coronavirus (SARS-CoV-2) and causes substantial morbidity and mortality. At the time the study was planned, there were no vaccines to prevent COVID-19 or infection with SARS-CoV or therapeutic agent with demonstrated efficacy as specific treatment for COVID-19.

Convalescent plasma (CP) from infected patients who have developed an immune response is likely to be an option for the treatment of patients with a variety of severe viral diseases. This would include patients in the most recent epidemics with coronaviruses, SARS1 in 2003 and MERS in 2012, and potentially as well patients in the current COVID-19 pandemic. Despite suggesting safety and potential efficacy, the available evidence has the major limitation of being based on predominantly low-quality uncontrolled studies [1]. Here we present a summary of the rationale and justification for conducting a multicenter, randomized clinical trial of CP therapy in COVID-19 hospitalized patients.

Passive immunotherapy involves the administration of antibodies against a given agent to a susceptible individual with the purpose of preventing or treating an infectious disease caused by that agent. Historically $\mathrm{CP}$ has been used in outbreaks of poliomyelitis, measles, mumps, influenza (1918 H1N1 and 2009-2010 H1N1), and 2013 Ebola [2]. In addition, although less readily available and requiring more complex manufacturing than $\mathrm{CP}$, conventional and hyperimmune immunoglobulins are used in clinical practice on a number of infections such as respiratory syncytial virus, hepatitis $B$, and others [3].

Currently, the only source of antibodies available for immediate use against SARS-CoV-2 is human CP. This is a readily available resource during an epidemic crisis even in low-income countries, as it uses the infrastructure and means developed for blood transfusions. In addition, as more individuals contract COVID-19 and recover, the number of potential donors will continue to increase in all areas where COVID-19 epidemic is present [4].

The experience with severe acute respiratory infections (SARI) caused by a coronavirus is rather recent in a number of epidemics in the twenty-first century. Human
CP was used in patients from both SARS-1 in 2003 and MERS in 2012. Overall, the experience showed that CP is safe and likely to reduce mortality in patients with coronavirus-related SARI. The largest study with 80 patients with SARS in Hong Kong in 2003 [5] and subsequent publications $[6,7]$ point out that earlier administration after symptom onset is more effective, particularly before day 14, prior to seroconversion in patients remaining PCR test positive.

From this background and rationale, we have developed this study with the objective of evaluating the safety and efficacy of $\mathrm{CP}$ in hospitalized adult patients with severe COVID-19. In the midst of a worldwide pandemic of SARS-CoV-2 and COVID-19, CP was hypothesized to represent a potential effective therapeutic option with a favorable safety profile for these patients.

\section{Objectives}

The trial objective is to evaluate the clinical efficacy and safety of Convalescent Plasma combined with standard of care (SOC) compared with SOC alone in adult patients with severe COVID-19.

\section{Study design}

This is a phase 2, parallel group, randomized, openlabel, controlled, superiority, multicenter clinical trial.

The protocol has been prepared in accordance with the SPIRIT (Standard Protocol Items: Recommendations for Interventional Trials) guidelines (Fig. 1).

Approximately 278 patients (139 per arm) with severe SARS-CoV-2 pneumonia will be enrolled at 32 centers. Individuals fulfilling selection criteria will be randomized to receive $\mathrm{CP}(+\mathrm{SOC})$ or $\mathrm{SOC}$ alone at a ratio of $1: 1$. Also, approximately 140-200 CP donors will be recruited.

The study has been planned with a sequential design. Interim analyses for safety monitoring and for comprehensive efficacy/futility will be conducted when $20 \%$, $40 \%, 60 \%$, and $80 \%$ of patients have been recruited and completed D15 primary endpoint assessment, or at the discretionary criteria of the Data Safety Monitoring Board (DSMB) when needed. 


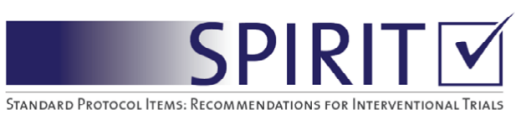

SPIRIT 2013 Checklist: Recommended items to address in a clinical trial protocol and related documents*

\begin{tabular}{|c|c|c|c|}
\hline Section/item & $\begin{array}{l}\text { Item } \\
\text { No }\end{array}$ & Description & $\begin{array}{l}\text { Addressed on } \\
\text { page number }\end{array}$ \\
\hline \multicolumn{4}{|c|}{ Administrative information } \\
\hline Title & 1 & $\begin{array}{l}\text { Descriptive title identifying the study design, population, interventions, and, if applicable, } \\
\text { trial acronym }\end{array}$ & 1,9 \\
\hline \multirow[t]{2}{*}{ Trial registration } & $2 a$ & Trial identifier and registry name. If not yet registered, name of intended registry & 1 \\
\hline & $2 \mathrm{~b}$ & All items from the World Health Organization Trial Registration Data Set & 1 \\
\hline Protocol version & 3 & Date and version identifier & 1 \\
\hline Funding & 4 & Sources and types of financial, material, and other support & 1 \\
\hline \multirow{4}{*}{$\begin{array}{l}\text { Roles and } \\
\text { responsibilities }\end{array}$} & $5 a$ & Names, affiliations, and roles of protocol contributors & $3-5$ \\
\hline & $5 b$ & Name and contact information for the trial sponsor & 3 \\
\hline & $5 c$ & $\begin{array}{l}\text { Role of study sponsor and funders, if any, in study design; collection, management, } \\
\text { analysis, and interpretation of data; writing of the report; and the decision to submit the } \\
\text { report for publication, including whether they will have ultimate authority over any of } \\
\text { these activities }\end{array}$ & $3-6$ \\
\hline & $5 d$ & $\begin{array}{l}\text { Composition, roles, and responsibilities of the coordinating centre, steering committee, } \\
\text { endpoint adjudication committee, data management team, and other individuals or } \\
\text { groups overseeing the trial, if applicable (see ltem 21a for data monitoring committee) }\end{array}$ & $3-6$ \\
\hline \multicolumn{4}{|l|}{ Introduction } \\
\hline \multirow[t]{2}{*}{$\begin{array}{l}\text { Background and } \\
\text { rationale }\end{array}$} & $6 a$ & $\begin{array}{l}\text { Description of research question and justification for undertaking the trial, including } \\
\text { summary of relevant studies (published and unpublished) examining benefits and harms } \\
\text { for each intervention }\end{array}$ & $15-17$ \\
\hline & $6 \mathrm{~b}$ & Explanation for choice of comparators & 20 \\
\hline Objectives & 7 & Specific objectives or hypotheses & 17 \\
\hline Trial design & 8 & $\begin{array}{l}\text { Description of trial design including type of trial (eg, parallel group, crossover, factorial, } \\
\text { single group), allocation ratio, and framework (eg, superiority, equivalence, non- } \\
\text { inferiority, exploratory) }\end{array}$ & 20 \\
\hline \multicolumn{4}{|c|}{ Methods: Participants, interventions, and outcomes } \\
\hline Study setting & 9 & $\begin{array}{l}\text { Description of study settings (eg, community clinic, academic hospital) and list of } \\
\text { countries where data will be collected. Reference to where list of study sites can be } \\
\text { obtained }\end{array}$ & 21 \\
\hline Eligibility criteria & 10 & $\begin{array}{l}\text { Inclusion and exclusion criteria for participants. If applicable, eligibility criteria for study } \\
\text { centres and individuals who will perform the interventions (eg, surgeons, } \\
\text { psychotherapists) }\end{array}$ & 21-22 \\
\hline \multirow[t]{4}{*}{ Interventions } & 11a & $\begin{array}{l}\text { Interventions for each group with sufficient detail to allow replication, including how and } \\
\text { when they will be administered }\end{array}$ & 20 \\
\hline & 11b & $\begin{array}{l}\text { Criteria for discontinuing or modifying allocated interventions for a given trial participant } \\
\text { (eg, drug dose change in response to harms, participant request, or } \\
\text { improving/worsening disease) }\end{array}$ & $\begin{array}{l}\mathrm{N} / \mathrm{A} \text {, this was a } \\
\text { single dose } \\
\text { intervention } \\
\text { study }\end{array}$ \\
\hline & $11 \mathrm{c}$ & $\begin{array}{l}\text { Strategies to improve adherence to intervention protocols, and any procedures for } \\
\text { monitoring adherence (eg, drug tablet return, laboratory tests) }\end{array}$ & $\begin{array}{l}\text { N/A, this was a } \\
\text { single dose } \\
\text { intervention } \\
\text { study }\end{array}$ \\
\hline & 11d & $\begin{array}{l}\text { Relevant concomitant care and interventions that are permitted or prohibited during the } \\
\text { trial }\end{array}$ & 25 \\
\hline Outcomes & 12 & $\begin{array}{l}\text { Primary, secondary, and other outcomes, including the specific measurement variable } \\
\text { (eg, systolic blood pressure), analysis metric (eg, change from baseline, final value, time } \\
\text { to event), method of aggregation (eg, median, proportion), and time point for each } \\
\text { outcome. Explanation of the clinical relevance of chosen efficacy and harm outcomes is } \\
\text { strongly recommended }\end{array}$ & $17-19$ \\
\hline
\end{tabular}

Fig. 1 SPIRIT 2013 Checklist: Recommended items to address in a clinical trial protocol and related documents*

\section{Methods: participants, interventions, and outcomes}

\section{Participating centers}

Study clinical sites included 33 public and private tertiary hospitals across the different regions in Spain. The only requirement for the participating study sites was a local research team that includes as co-principal investigators both a hematologist and an infectious diseases or internal medicine investigator, together with participation in the trial of the reference Transfusion Center. Transfusion services or centers at the hospitals or Autonomous Communities and National Army Transfusion Center are essential participants in this trial. ISCIII (Centro Nacional de Microbiología, Instituto de Salud Carlos III) centralized the antibodies and PCR microbiological testing from patients and donors (Additional file 1). 


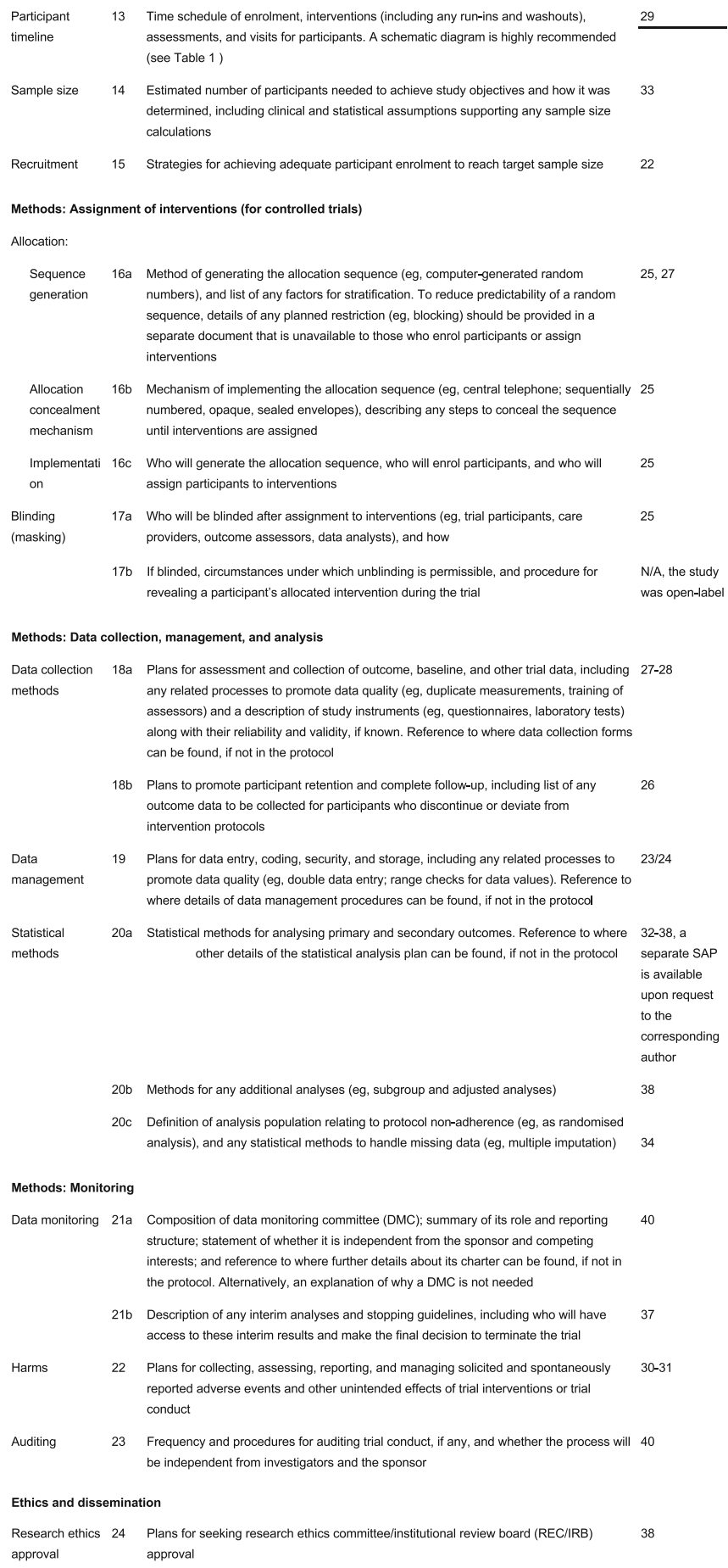

Fig. 1 SPIRIT 2013 Checklist: Recommended items to address in a clinical trial protocol and related documents*

\section{Eligibility criteria}

\section{Eligibility criteria for patients}

Inclusion criteria are as follows:

1. Written informed consent prior to performing study procedures. Witnessed oral consent will be accepted in order to avoid paper handling, which increased the risk of transmission of the infection. Written consent by patient or representatives will be obtained as soon as possible.

2. Male or female adult patient $\geq 18$ years of age at the time of enrolment.

3. Laboratory-confirmed SARS-CoV-2 infection as determined by PCR in naso/oropharyngeal swabs 


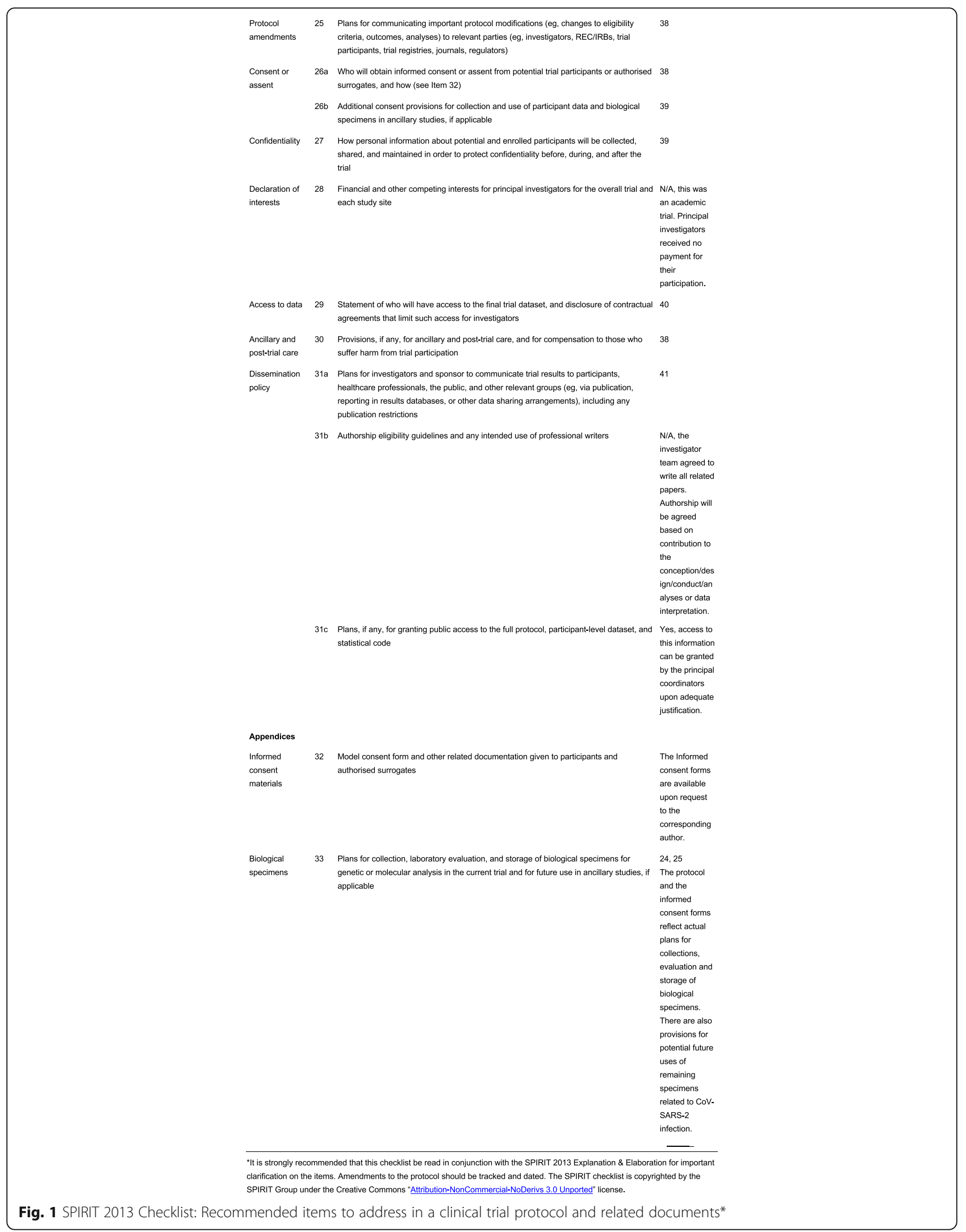


or any other relevant specimen obtained in the ongoing COVID-19 symptomatic period. Alternative tests (i.e., rapid antigenic tests) are also acceptable as laboratory confirmation if their adequate specificity has been accepted by the sponsor.

4. Patients requiring hospitalization for COVID-19 without mechanical ventilation (invasive or noninvasive) or high flow oxygen devices and at least one of the following:

- Radiographic evidence of pulmonary infiltrates by imaging (chest X-ray, CT scan, etc.) OR

- Clinical assessment (evidence of rales/crackles on exam) and $\mathrm{SpO} 2 \leq 94 \%$ on room air that requires supplemental oxygen.

5. No more than 12 days between the onset of symptoms (fever or cough) and treatment administration day. Following 31 August amendment, only patients with no more than 7 days between the onset of symptoms and treatment administration day are allowed for inclusion.

\section{Exclusion criteria are as follows:}

1. Requiring mechanical ventilation (invasive or noninvasive) or high flow oxygen devices at screening.

2. More than 12 days since symptoms (fever or cough) onset.

3. Participation in any other clinical trial of an experimental treatment for COVID-19.

4. In the opinion of the clinical team, progression to death is imminent and inevitable within the next $24 \mathrm{~h}$, irrespective of the provision of treatments.

5. Any incompatibility or allergy to the administration of human plasma.

6. Stage 4 severe chronic kidney disease or requiring dialysis (i.e., eGFR < 30).

\section{Eligibility criteria for donors}

Inclusion criteria are as follows:

1. Subjects willing and able to provide written informed consent.

2. Fulfilling all the current requirements to be a plasma apheresis donor according to the regulations for donation of blood products (European Guidelines and RD 1088/2005 in Spain).

3. Absence of COVID-19 symptoms within the last 14 days.

4. Anti SARS-CoV-2 IgG antibodies detectable in peripheral blood.

5. $\geq 18$ years of age at time of donation.
6. Weight $>50 \mathrm{~kg}$ and good vein access are standard criteria, for which exceptions could be considered according to the criteria of the blood bank and hematologist.

\section{Exclusion criteria are as follows:}

1. Plasmapheresis in the previous 7 days.

2. Whole blood donation in the previous 30 days.

3. Donation of more than $25 \mathrm{l}$ of plasma in the previous 12 months.

\section{Informed consent}

Investigators will obtain the subject's informed consent in accordance with Spanish Law 14/2007 on Biomedical Investigation and the internationally ethical accepted guidelines.

Patients will receive a concise and focused presentation of key information about the clinical trial, orally, and a written informed consent form will be handled to the patient. Due to paper handling limitation in COVID wards, oral witnessed consent will be accepted before entering into the trial, with written documentation in the patient clinical record. If possible, written consent form will be obtained from the patient himself or acceptable representatives, at a later time.

Donors will receive concise information about the clinical trial and will give written informed consent before donating convalescent plasma.

\section{Additional consent provisions}

The consent form includes provisions for research data and residual samples to be stored for future scientific research on COVID-19. These future studies will be previously evaluated by a Research Ethics Committee and will comply with the applicable ethical and legal requirements.

\section{Study interventions Intervention description}

All trial participants will receive SOC for COVID-19. The control arm is SOC for COVID-19. In the treatment arm, patients will also receive intravenous pathogenreduced CP from patients recovered from COVID-19 (designated as donors) as add-on therapy to SOC.

In the current status of a worldwide pandemic for which we have no approved vaccines or drugs, for the purpose of this trial SOC will include any medicinal products being used in clinical practice (e.g., lopinavir/ritonavir; darunavir/cobicistat; hydroxy/chloroquine, tocilizumab, remdesivir, allowed as SOC when its use outside clinical trials was permitted), other than those used as part of another clinical trial.

Donor assessment, pathogen-reduced plasma collection, and production will be performed by hospital 
Transfusion Services and Regional Transfusion Centers. Local organization will be adapted to the existing structure at the regional level.

\section{Criteria for discontinuing or modifying allocated interventions}

A patient may be removed from the study treatment for the reasons mentioned below, although whenever possible the patient should be followed regardless of their protocol adherence as per the efficacy and safety evaluations:

- Patient withdraws consent or requests discontinuation from the study for any reason

- Termination of the study.

- Lost to follow-up.

Patients who withdraw from this study or are lost to follow-up after signing the informed consent form (ICF) will not be replaced. The reason for patient discontinuation from the study will be recorded on the appropriate case report form.

\section{Strategies to improve adherence to interventions}

This item is not applicable, since the active agent is administered intravenously by health care professionals in a single-dose administration.

\section{Relevant concomitant care permitted or prohibited during the trial}

This study seeks to investigate the effects of CP in addition to standard of care. All concomitant care and interventions are permitted other than concomitant administration of any other experimental treatment.

\section{Provisions for post-trial care}

No special arrangements for post-trial care are anticipated.

\section{Outcomes}

\section{Primary outcome measure}

The primary outcome measure is the proportion of patients that progress to category 5, 6, or 7 (hospitalized severe disease or death categories) on the 7point ordinal scale recommended in the Master Protocol of the WHO R\&D Blueprint expert group, at day 15 (Table 1).

\section{Secondary outcome measures}

The secondary outcome measures include the following:

-Ordinal scale (see 7-point ordinal scale above):
Table 1 Ordinal scale for illness severity

1. Not hospitalized, no limitation of activities

2. Not hospitalized, limitation of activities

3. Hospitalized, not requiring supplemental oxygen

4. Hospitalized, requiring supplemental oxygen by mask or nasal prongs

5. Hospitalized, non-invasive ventilation or high flow oxygen

6. Hospitalized, intubation and mechanical ventilation or ECMO

7. Death

- Status at day 30

- Time to category 5, 6, or 7 of the ordinal scale.

- Time to an improvement of one category from admission on the ordinal scale.

- Time to first deterioration

- Mean change in the ranking on an ordinal scale from baseline to days 3, 5, 8, 11, 15, 29, and 60 .

-Ordinal scale of 11 points (Additional file 2):

- Mean change in the ranking on an ordinal scale from baseline to days 3, 5, 8, 11, 15, 29, and 60

- Status at day 15 and 30

- Mortality rate (all cause) at day 15 , at day 29, and at day 60.

- Oxygenation-free days in the first 28 days (day 29).

- Ventilator-free days in the first 28 days (day 29).

- Duration of hospitalization (days).

- Incidence of thrombotic arterial events.

- Incidence of thrombotic venous events.

- Rate of rehospitalizations.

- Serum level of CRP, lymphocyte count, LDH, D Dimer, IL-6, coagulation tests at baseline and at days $3,5,8,11,15,29$, and 60 .

- Safety assessments of CP + SOC as compared to SOC alone through day 60 considering cumulative incidence of serious adverse events (SAEs), cumulative incidence of Grade 3 and 4 adverse events (AEs), and infusion-related adverse reactions.

Exploratory outcomes include the following virology and immunology assessments

a) Qualitative and quantitative RT-PCR for SARSCoV-2 in nasopharyngeal/oropharyngeal swabs at baseline and at hospital discharge;

b) Qualitative and quantitative RT-PCR for SARSCoV-2 in blood on days 3, 5, 8, 11, 15, 29, and 60 (while hospitalized), until two of them are negative consecutively; 
c) Quantitative anti SARS-CoV-2 antibodies level at baseline and on days $8,15,29$, and 60 (while hospitalized);

d) Neutralizing antibodies study in plasma donors and a subset of patients at baseline.

\section{Participant timelines}

The schedule of interventions and visits can be found in Fig. 2.

\section{Sample size}

We propose an open-label, standard of care controlled, randomized (1:1 ratio) clinical trial with stopping boundaries for efficacy and futility at $20,40,60$, and $80 \%$ of the final sample size. The statistical design including the sample size and stopping have been calculated using the East validated software v6.5 by Cytel Inc. The stopping boundaries for superiority and inferiority have been calculated using the rho family spending functions.

The primary outcome measure is the proportion of patients that progress to category 5, 6, or 7 (hospitalized with severe disease or death categories of the 7-point ordinal scale), at day 15 . With this design, 278 patients (139 per arm) will be required assuming $20 \%$ rate in the control group and an absolute reduction of $10 \%(10 \%$ rate in experimental group), with $80 \%$ statistical power and a $2.5 \%$ one-sided alpha level (5\% two-sided). Likewise, approximately 140-200 CP donors will be needed.

With regard to the final sample size, it is predefined that a sample size recalculation will be put in place when $60 \%$ of the patients with assessed events at day 15 are available and the 3 rd analyses are conducted.

\section{Recruitment}

Patients with COVID-19 will be recruited at the participating clinical sites.

\section{Assignment of interventions: allocations Sequence generation}

Randomization among the two arms will be 1:1 and will be stratified per center.

The randomization process has been developed using the RERAND system integrated within the RDC Onsite ECRF system based on Oracle.

\section{Concealment mechanism}

This open-label trial will use blind randomization of patients in a 1:1 ratio to $\mathrm{CP}(+\mathrm{SOC})$ or $\mathrm{SOC}$ alone through a centralized system embedded in the eCRF (Oracle Clinical). Baseline clinical data will be entered in the eCRF before the patient can be randomly assigned via the eCRF at 1:1 ratio to receive standard of care with or without $\mathrm{CP}$ as add-on therapy. The system will automatically notify the assigned treatment arm at the eCRF screen and will send a confirmatory message at the email with the randomization information.

\section{Implementation}

Patients in the treatment arm will receive a single unit of CP (250-300 mL) after randomization on day 1 under control of the patient's clinician and the Transfusion Service's hematologist of the hospital.

Pathogen-reduced plasma bags will be frozen below $25{ }^{\circ} \mathrm{C}$ and stored and shipped following European guidelines for blood components storage (36 months below $25^{\circ} \mathrm{C}$ or 3 months below $-18^{\circ} \mathrm{C}$ ). For transport, plasma bags must be kept frozen. No special containers are needed if plasma units are kept frozen at the delivery.

\section{Assignment of interventions: blinding}

This is an open-label study. To reduce treatment bias, the eCRF did not allow randomizing a subject until sufficient clinical information from the subject was already included, reducing the possibility that a given subject could be re-randomized. Additional measures were implemented to reduce assessment bias, e.g., clinical information was collected, and daily clinical decisions were taken by the medical staff at each study center, that in most cases were not part of the research team. The primary endpoint was restricted to WHO scores $\geq 5$ in an attempt to not only focus on relevant outcomes, but also to increase the robustness of the endpoint; although some scores are subjected to clinical judgment, these are hardly influenced by the clinical trial participation and/ or treatment assignment.

\section{Data collection and management}

Plans for assessment and collections of outcomes

Investigators are responsible for assessment and collection of outcomes, baseline, and other trial data. Data will be entered in the CRF by delegated team members and will be monitored by the clinical research associated. Subjects will be assessed daily while hospitalized. Patients discharged before the end of follow-up will be regularly phoned or asked to attend study visits. NP/OP swabs for virus analysis and blood samples for serological analysis will be sent to a central laboratory, where test will be performed according to laboratory standard operating procedures.

\section{Plans to promote participants retention and complete follow-up}

Patients are free to withdraw from participation in the study at any time. The decision must be communicated and reviewed by investigators. Staff at study sites should explain to these subjects the importance of staying in the study for the full duration of follow-up of this trial for safety reasons. The reason for patient 


\begin{tabular}{|c|c|c|c|c|c|c|c|}
\hline VISITS & Screening ${ }^{1}$ & Baseline $^{1}$ & \multicolumn{2}{|c|}{ Follow-Up VISITS ${ }^{4}$} & Day15 $^{4}$ & Day29 ${ }^{4}$ & $\begin{array}{l}\text { Day60 } \\
\text { (End of } \\
\text { study) }\end{array}$ \\
\hline Day +/- Window & -3 to 1 & 1 & \multicolumn{2}{|c|}{$\begin{array}{l}\text { Daily until hospital } \\
\text { discharge }\end{array}$} & \pm 2 & \pm 3 & \pm 3 \\
\hline \multicolumn{8}{|l|}{ ASSESSMENTS/PROCEDURES } \\
\hline Informed consent & $x$ & & & & & & \\
\hline Inclusion and exclusion criteria & $x$ & $x$ & & & & & \\
\hline Demographics \& Medical History & $x$ & & & & & & \\
\hline SARS-CoV-2 PCR or antigen test & $x^{2}$ & & & & & & \\
\hline Rx Thorax & $\mathrm{X}^{7}$ & & & & & & \\
\hline Randomization & & $x$ & & & & & \\
\hline $\begin{array}{l}\text { Administration Convalescent } \\
\text { Plasma }\end{array}$ & & $x$ & & & & & \\
\hline Clinical data collection & & $x$ & \multicolumn{2}{|c|}{$\begin{array}{l}\text { Daily until hospital } \\
\text { discharge }\end{array}$} & $x$ & $x$ & $x$ \\
\hline Vital signs: $\mathrm{SpO}_{2} / \mathrm{T}^{\mathrm{a}}$ & $x$ & $x$ & \multicolumn{2}{|c|}{$\begin{array}{l}\text { Daily until hospital } \\
\text { discharge }\end{array}$} & $x$ & $x$ & $x$ \\
\hline Oxygen requirement & & $x$ & \multicolumn{2}{|c|}{$\begin{array}{l}\text { Daily until hospital } \\
\text { discharge }\end{array}$} & $x$ & $x$ & \\
\hline Mechanical ventilator requirement & & $x$ & \multicolumn{2}{|c|}{$\begin{array}{l}\text { Daily until hospital } \\
\text { discharge }\end{array}$} & $x$ & $x$ & \\
\hline Mortality & & & \multicolumn{2}{|c|}{$\begin{array}{l}\text { Daily until hospital } \\
\text { discharge }\end{array}$} & $x$ & $x$ & \\
\hline $\begin{array}{l}\text { Concomitant medication } \\
\text { (Only related with COVID-19) }\end{array}$ & & $x$ & \multicolumn{2}{|c|}{$\begin{array}{l}\text { Daily until hospital } \\
\text { discharge }\end{array}$} & $x$ & $x$ & $x$ \\
\hline AE /SAE (eCRF reporting) & & \multicolumn{3}{|c|}{ Daily until hospital discharge } & $x$ & $x$ & $x$ \\
\hline Blood samples (ABO group) & & $x$ & & & & & \\
\hline $\begin{array}{l}\text { Routine blood samples } \\
\text { (hematology and chemistry) }^{3}\end{array}$ & & $x$ & \multicolumn{2}{|c|}{$\begin{array}{c}(3,5,8 \text { and } 11 \\
\text { days })^{5}\end{array}$} & $x^{5}$ & $x^{5}$ & $x^{5}$ \\
\hline Blood for PCR SARS-CoV- ${ }^{6}$ & & $x$ & \multicolumn{2}{|c|}{$\begin{array}{l}(3,5,8 \text { and } \\
11 \text { days })^{5,6}\end{array}$} & $x^{5,6}$ & $x^{5,6}$ & $x^{5,6}$ \\
\hline Blood for antibodies determination & & $x$ & \multicolumn{5}{|c|}{$(8,15,29 \text { and } 60 \text { days })^{5}$} \\
\hline Naso/Oropharyngeal swab & & $\mathrm{x}^{2}$ & \multicolumn{5}{|c|}{ At discharge } \\
\hline \multicolumn{8}{|c|}{$\begin{array}{l}\text { 1. This visit can be done at the same time as the screening visit } \\
\text { 2. Positive PCR or COVID-19 antigen test (accepted by the sponsor), in naso/oropharyngeal swabs or any other } \\
\text { relevant specimen is needed prior to randomization. If inclusion is based on a previous local swab, a new } \\
\text { basal swab to be sent to CNM-ISCIII will be needed. If previous SARS-CoV-2 test + is not available and the } \\
\text { swab is obtained as a screening procedure, the basal swab could be obtained at the same time, taking into } \\
\text { account that the basal swab HAS TO BE SENT to the CNM-ISCIII central lab. } \\
\text { 3. Haematology, chemistry, ferritin, CRP, LDH, IL-6, coagulation, D-dimer, CPK, platelets and troponine. } \\
\text { 4. These visits may be conducted by phone. In this case, blood samples and vital signs will not be available. } \\
\text { 5. Only during hospitalization. } \\
\text { 6. If two consecutive negative results are obtained, no more PCR tests needed. } \\
\text { 7. RX obtained during the ongoing COVID-19 symptomatic period (maximum } 7 \text { days old). }\end{array}$} \\
\hline
\end{tabular}

Fig. 2 schedule of study procedures 
discontinuation from the study will be recorded on the appropriate case report form.

In cases where that a patient becomes lost to followup, attempts to contact the patient should be made and documented in the patient's medical records. Patients who withdraw from this study or are lost to follow-up after signing the informed consent form (ICF) and receiving the study product, will not be replaced.

\section{Data management}

Data will be examined for compliance with the trial protocol by the monitor and the data manager. Deviations will be sent to the project statistician to plan listings for the data review (DR). The objective is to carry out the population selection and definition of the final study populations as well as a preliminary assessment of the quality of the trial data.

All data will be recorded using the defined CRF guidelines for this trial. Also, the blood bank software in each hospital Transfusion Service will be used for recording the plasma units transfused.

\section{Confidentiality}

Subject confidentiality is strictly held in trust by the participating investigators, their staff, and the sponsor and their agents. This confidentiality is extended to cover clinical information relating to subjects, test results of biological samples, and all other information generated during participation in the study. All study data and research specimens that leave the site (including any electronic transmission of data) will be identified only by a coded number that is linked to a subject through a code key maintained at the clinical site. All source records including electronic data will be stored in secured systems.

No identifiable information concerning subjects in the study will be released to any unauthorized third party. Subject confidentiality will be maintained when study results are published or discussed in conferences. The study monitor, other authorized representatives of the sponsor, representatives of the Research Ethics Committees (RECs), and/or regulatory agencies may inspect all documents and records required to be maintained by the investigator, including but not limited to medical records (office, clinic, or hospital) for the subjects in this study. The clinical study site will permit access to such records.

\section{Plans for collection, laboratory evaluation, and storage of biological specimens for genetic or molecular analysis in this trial/future use}

Samples will be collected by investigators or designees. After that, handling, labeling, processing, storage, and/or shipping according to protocol will be performed and the samples will ultimately be sent to the central laboratory.

The Sponsor and the center may use data and samples for future research projects related with COVID-19, taking the appropriate measures to ensure the protection of their privacy and will not allow their encrypted data to cross with other databases that could allow their identification. Any future studies will be previously evaluated by a Research Ethics Committee and will comply with the applicable ethical and legal requirements.

\section{Statistical methods}

\section{Statistical methods for primary and secondary outcomes}

A detailed Statistical Analysis Plan (SAP) agreed upon by the CT Executive Board and the Project Statistician will be available early during the recruitment phase. This SAP will follow the general regulatory recommendations given in the ICHE9 (CPMP/ICH /363/96) guidance, as well as other specific guidance on methodological and statistical issues [8].

Likewise, it will stick to the recommendations given by the consensus documents of the scientific journals to improve reliability and value of medical research literature by promoting transparent and accurate reporting of clinical research studies.

The proportion of patients with failure, defined as category 5,6 , or 7 of the 7 -point ordinal scale at day 15 , will be estimated using a log-binomial regression model including stratification variables. In the unexpected event that the model does not fit, the Poisson regression model with long-link and robust variance estimator will be used instead.

Binary efficacy and safety outcomes will be analyzed as described for the primary endpoint.

Kaplan-Meier model will be used to analyze survival endpoints (TTF and OS). In all these analyses, in addition to the Kaplan-Meier curve, median, Q1, Q3, and their corresponding 95\% CI, number of events, and censored case distribution will be shown. Group comparisons will be done using the (stratified) log-rank test and the (stratified) hazard ratios (HR) $(95 \% \mathrm{CI})$ from the Cox model.

Interim analyses Interim analyses for comprehensive efficacy (or futility) and safety data monitoring analyses will be conducted when $20,40,60$, and $80 \%$ of patients have been recruited and completed day 15 assessment, or at the discretionary criteria of the DSMB, when needed. The study may be stopped prematurely if either the efficacy or the futility boundaries are crossed. The boundaries will be adapted to the actual information rates in each inspection using the rho family spending functions $($ rho $=7)$ implemented in the East validated software v6.5 (or later releases) by Cytel Inc.. 
Ad hoc reviews will be undertaken at any time if there are other specific safety concerns. The study will not stop enrolment awaiting these DSMB reviews, though the DSMB may recommend temporary or permanent cessation of enrolment based on their safety reviews.

Methods for additional analyses (e.g., subgroups) Subgroup analyses will be performed to assess the impact on efficacy of two different key factors, i.e., the level of neutralizing antibodies in the administered plasma and the timing of the disease, i.e., early or late stages considering 7 days as cut-off, viral load, and IgG or IgM. The following strategy will be conducted before splitting the analysis into subgroups: test of the overall treatment effect, test of the treatment-by-subgroup interaction at the $10 \%$ level of significance, or test of the treatment effect in each subgroup category.

Methods to handle missing data In principle, the rate of missing data is estimated to be very low due to the type of endpoint, easily available with a fast-clinical assessment, so no impact on the primary analysis is expected. In any case, a very conservative strategy will be implemented consisting of imputing any missing data or other binary efficacy secondary outcomes will be considered to failures, irrespectively to the reason for missingness. With regard to the continuous variables, mixed models [9-11] are robust to the presence of missing at random (MAR) and conduct the analysis with all participants despite the presence of missingness. Of note, this method calculates the estimations based on the variance-covariance structure but without any formal imputations.

Plans to give access to the full protocol, participantlevel data, and statistical code These plans are not yet in place.

\section{Oversight and monitoring Composition of the coordinating center and trial steering committee}

- Coordinating center The study is led by the Hospital Universitario Puerta de Hierro Majadahonda.

- Trial steering committee The trial steering committee consists of the following members:

- Dr. Rafael Duarte, Hematology and Hemotherapy Department. Hospital Universitario Puerta de Hierro Majadahonda

- Dr. Cristina Avendaño Solà, Clinical Pharmacology Department. Hospital Universitario Puerta de Hierro Majadahonda
- Dr. Antonio Ramos-Martínez, Internal Medicine Department. Infectious diseases Unit. Hospital Universitario Puerta de Hierro Majadahonda

- Dr. José Luis Bueno, Hemotherapy and Apheresis Units. Hematology and Hemotherapy Department. Hospital Universitario Puerta de Hierro Majadahonda

- Dr. Elena Múñez, Internal Medicine Department. Infectious diseases unit. Hospital Universitario Puerta de Hierro Majadahonda

- Dr. Belén Ruiz-Antorán, Clinical Pharmacology Department. Hospital Universitario Puerta de Hierro Majadahonda

- Dr. Rosa Malo de Molina, Servicio de Pneumology. Hospital Universitario Puerta de Hierro Majadahonda

- Dr. Ferrán Torres, Clinical Pharmacology Department. Hospital Clínic Barcelona. Medical Statistics core facility - IDIBAPS.

- Dr. Inmaculada Casas Flecha, Flu and Respiratory Virus Unit. Centro Nacional de Microbiología, Instituto de Salud Carlos III

\section{Trial monitoring}

The Spanish Clinical Research Network (SCReN) is responsible for project management, regulatory compliance and trial monitoring.

\section{Data management team}

PIVOTAL is the CRO responsible for data management, preparation of the eCRD, quality assurance, and preparation of the SAP.

\section{Composition of the data monitoring committee, its role and reporting structure}

The independent DSMB in this study is responsible for reviewing the reports regarding the safety and efficacy of the study patients protocol adherence and making recommendations to continue or terminate the study or to modify the sample size of the basis of the results from the interim analysis. The DSMB members are all independent of the sponsor and have no financial or other conflict of interest.

\section{Adverse event reporting}

Serious adverse events (SAEs) and grade 3 or 4 adverse events will be collected from the time of informed consent to day 29. SAEs will be followed up until the SAE has subsided, returned to baseline, or is stable. Infusionrelated adverse reactions will be recorded within $24 \mathrm{~h}$ after the end of plasma administration by a trained Hemovigilance nurse or physician, according to the Active $24 \mathrm{~h}$ quarantine Hemovigilance Program (HEMA CUA). 
Investigators will be instructed to actively monitor the occurrence of prespecified adverse events of special interest: TRALI (transfusion-related acute lung injury), ADE (antibody-dependent enhancement of infection, and TACO (transfusion-associated cardiac overload).

\section{Plans for auditing trial conduct}

Monitoring for this study will be performed by the sponsor and SCReN. Monitoring online visits will include, but not limited to, review of regulatory files, accountability records, CRFs, ICFs, medical and laboratory reports, site study intervention storage records, training records, and protocol and GCP compliance.

On-site and off-site monitoring, central review of data collection, and remote source data verification will be allowed according to EMA and AEMPS guidance/guidelines on the management of clinical trials during the COVID-19 (coronavirus) pandemic.

\section{Plans for communicating important protocol amendments to relevant parties (e.g., trial participants, ethical committees)}

During the trial, any amendments to the protocol or consent materials will be approved by the REC before they are implemented.

\section{Dissemination plans}

Following completion of the study, the results will be published in a scientific journal. Nevertheless, due to the critical need of results during the current epidemic COVID-19 crisis, preliminary results will be released by the sponsor to the Health Authorities.

\section{Ethical and regulatory}

The clinical study will be conducted in accordance with the relevant national and international good clinical practice (GCP) guidelines, and the Declaration of Helsinki, each in the applicable version. The study protocol and the donors and the patients' written informed consent were submitted to and approved by the Research Ethics Committee of Hospital Puerta de Hierro Majadahonda on March 23th, 2020.

\section{Discussion}

COVID-19 is a respiratory disease caused by a novel coronavirus (SARS-CoV-2) and causes substantial morbidity and mortality. At the time the study protocol was designed, there were no vaccines to prevent COVID-19 or infection with SARS-CoV-2 or therapeutic agent to treat COVID-19. Indeed, as in any other health emergency, there was an enormous pressure to find cures and stop COVID-19 epidemic. This has prompted the extensive use of unproven treatments either on a compassionate use basis, in observational studies, or even in small clinical trials, but these do not provide the required level of evidence to solve the existing uncertainties. Thus, there was a substantial need to conduct appropriately powered randomized controlled studies in order to generate a reliable and conclusive evidence regarding the benefits and risks of these therapies.

In this scenario, convalescent plasma was hypothesized to be a potential therapeutic option given its extensively recognized immunomodulatory and anti-inflammatory effects. However, the actual benefits and risks of the intervention remain to be established, particularly in this novel condition. Currently, there are over 250 studies registered in ClinicalTrials.gov about convalescent plasma as a treatment for respiratory disease caused by COVID-19 all over the world, most of which are noncontrolled clinical studies that are ongoing. Therefore, in accordance with the WHO Blood Regulators Network position statement [12] and our commitment to generate compelling evidence, we planned to conduct the proposed randomized and controlled trial to establish the effectiveness and safety of convalescent plasma from disease survivors in the treatment of patients with severe COVID-19 pneumonia.

This clinical trial is designed to evaluate passive immunotherapy with convalescent plasma for the treatment of adult patients hospitalized with non-severe COVID-19. With regard to the regulatory aspect, convalescent plasma from a single donor is not considered a medicinal product but rather it is subject to the regulation applicable to blood transfusions at the EU level. Subject to demonstration of its efficacy, it was considered that $\mathrm{CP}$ would constitute a universally accessible treatment option given that it relies entirely on already existing transfusions systems and technical requirements that are already established in every country. This makes CP a particularly attractive potential therapeutic option; even more considering that it is possible that the results of this study will contribute to the establishment of clinical recommendations to treat similar conditions.

The study was designed as an open-label study due to implications of a sham transfusion in the context of COVID-19 pandemic. The study was also designed as a controlled clinical trial. Initially, a three-treatment arm study including no experimental treatment (only SOC), unspecific standard plasma (plus SOC), and $\mathrm{CP}(+\mathrm{SOC})$ was considered. The aim was to demonstrate that any potential benefit from CP treatment was due to the presence of neutralizing antibodies and any other related cytokines released in a successful immune response and not just to the existing non-specific immune components in SARS-CoV-2 non-exposed donors. The inclusion of a third treatment arm with unspecific plasma might have also allowed ruling out any potential 
deleterious effect of transfusing an enriched plasma to a subject developing his/her own immune response. Nevertheless, a three-treatment arms trial was deemed unrealistic due to practical reasons and also to the wellknown adverse events related to blood component transfusion, some of them even fatal, specially transfusionrelated acute lung injury (TRALI).

Concerning the study population, a potential role of immunotherapy across the spectrum of COVID-19 disease has been suggested and several studies are ongoing to test different hypotheses. When defining the study population for our study, we considered that the best candidates for passive immunotherapy would be patients hospitalized due to COVID-19 pneumonia, thus in need for treatment, but early in the course of the disease when a primary immune response is not yet established. As a result, only patients requiring hospitalization for COVID-9 pneumonia without mechanical ventilation (invasive or non-invasive) or high flow oxygen devices, and with no more than 12 days between the onset of symptoms (fever or cough) and treatment administration day were allowed to enroll. It was believed that this early intervention would allow patients to benefit from the immune response of a subject who had developed a successful one, which might help clearing SARS-CoV-2 virus and to prevent progression to a more severe condition. On the other hand, patients requiring ventilation (mechanical or non-mechanical) were excluded given that they are less likely to respond to passive immunotherapy and more likely to develop ADE (antibodydependent enhancement of disease), a worrisome complication seen previously in other viral infection [13] where a high specific antibody infusion could trigger a severe life-threatening immune response.

With regard to convalescent plasma donor's selection, processing, and storage, it has been performed following the European and Spanish guidelines (RD 1088/2005) for standard plasma donation, including a pathogen reduction (PR) treatment. Lack of process control and standardization has been raised as one of the reasons why clinical trials have failed so far. We will select $\mathrm{CP}$ with anti SARS-CoV-2 IgG antibodies using a validated enzyme-linked immunosorbent assay (ELISA), with an IgG amount above a standardized cut-off. These assays have been performed at the trial central laboratory. No previous determination for neutralizing antibody titers will be used to select $\mathrm{CP}$ in our trial, as this would be the norm in the use of $\mathrm{CP}$ in the midst of a pandemic. Titration of neutralizing antibodies in the administered plasma will be subsequently performed and its relation to the outcomes could provide useful information about efficacy and safety of CP.

An important issue in our trial is that we selected our CP donors from recovered mild COVID-19 conditions.
Although patients recovered from severe COVID-19 was not an exclusion criteria for donors in our trial, we chose mild donors based in two assumptions: first, severe patients could have a high anti SARS-CoV-2 IgG titer, but low neutralizing antibody titers; second, high neutralizing antibodies titers in $\mathrm{CP}$ could increase the risk of ADE (antibody-dependent enhancement of disease). Thus, our donor selection strategy is aimed to select $\mathrm{CP}$ with medium levels of antibody titers, which is thought to provide a fair balance of efficacy and safety.

$\mathrm{CP}$ will be administered as an add-on to the standard of care as defined in each study center. Based on the clinical practice recommendations in place at the time the study was designed, the standard of care could be based on any of the following: lopinavir/ritonavir, darunavir/cobicistat, hydroxy/chloroquine, tocilizumab. Remdesivir was added to standard of care once it was available outside clinical trials. Demonstration of efficacy in this context is particularly challenging, but ethical and feasibility issues were prioritized.

Defining the primary endpoint posed additional challenges given the existing uncertainties around the natural course of the disease, and the need to balance hard clinically relevant outcomes against outcomes that might occur earlier. The ordinal scale recommended by the WHO for clinical trials in COVID-19 was finally selected, as it was considered to provide a reasonable balance between these two criteria, while facilitating any external comparison with similar clinical trial outcomes. We also include a range of secondary endpoints to assess the scope of the disease and the potential benefits/ risks of treatment in a broader spectrum, in an attempt to show consistency and the robustness of any potential effect shown in the primary endpoint.

Finally, to overcome practical difficulties due to paper handling limitations at COVID wards but to still comply with ethical requirements applied to clinical research, oral witnessed consent will be accepted before entering the trial. Written consent form will be obtained from the patient himself or acceptable representatives as soon as possible.

\section{Conclusion}

Due to the existing uncertainties on the potential role of convalescent plasma in adult patients with severe COVID-19 pneumonia, we consider that the publication of the study protocol will help other researchers to understand the rationale behind our clinical trial design and may contribute to define future research strategies with CP in the field of COVID-19 or other viral diseases.

\section{Trial status}

Patient's recruitment started on 4 April 2020, under conPlas-19 Protocol version 2.2 as of 22 April 2020. Donor's recruitment started on 2 April 2020. As of 10 July 
2020, patients' recruitment was temporarily interrupted awaiting some study modifications. By 31 August, the study design was amended to restrict the inclusion to patients with no more than 7 days of symptoms, and the follow-up was extended up to 60 days. ConPlas-19 Protocol version 3.3 as of 30 September 2020 is now in place, allowing the use of rapid antigenic tests as laboratory confirmation test for SARS-CoV-2 infection.. It is anticipated that recruitment will be complete by end of 2020.

\section{Supplementary Information}

The online version contains supplementary material available at https://doi. org/10.1186/s13063-020-05011-9.

Additional file 1. Clinical Trial sites and Transfusion centers.

Additional file 2. Eleven Point Ordinal Scale.

\section{Acknowledgements}

Not applicable.

\section{Authors' contributions}

CAS, RDP, JLB, BRA, FT, EM, ARM, AFC, IC, CPH did the literature search and conceived the study design. CAS acted as the study sponsor and principal investigator. RDP acted as principal investigator. ARM also acted as clinical trial national coordinator. JLB acted as plasma production coordinator. AVI also acted as project manager. ISD was crucial for trial organization and execution and revised the manuscript. EDS, ASL, and BRA drafted the manuscript, made the tables and figures, and had final approval of the manuscript. All authors critically revised the manuscript. The authors read and approved the final manuscript.

\section{Authors' information}

Not applicable

\section{Funding}

This research is funded by the Government of Spain, Ministry of Science and Innovation, Instituto de Salud Carlos III, grant $n^{\circ}$ COV20/00072 (Royal DecreeLaw 8/2020, of 17 March, on urgent extraordinary measures to deal with the economic and social impact of COVID-19), co-financed by the European Regional Development Fund (FEDER) "A way to make Europe," and supported by SCReN (Spanish Clinical Research Network), ISCIII, project PT17/0017/0009. The funding institutions do not have any role in the design of the study, data collection, analysis, or interpretation of data, nor in writing the manuscript.

\section{Availability of data and materials}

The datasets generated and/or analyzed during the current study will be made available. The corresponding authors will evaluate any request for data sharing and will consult with the steering committee after the publication of the main results. Requests can be sent to cavendano@salud.madrid.org.

\section{Ethics approval and consent to participate}

The study protocol and the donors and the patients' informed consent forms were submitted to and approved by the Research Ethics Committee of Hospital Puerta de Hierro Majadahonda on March 23, 2020 (REC number PI 57-20).

I herewith certify that this trial has received ethical approval from the appropriate ethical committee as described above. Consent from participants to participate in the study will be obtained before any study procedure.

\section{Author details}

Clinical Pharmacology Department, Hospital Universitario Puerta de Hierro Majadahonda, Instituto de Investigación Sanitaria Puerta de Hierro - Segovia de Arana, c/ Manuel de Falla 1, 28222 Madrid, Spain. ${ }^{2}$ Hemotherapy \& Apheresis Units, Hematology and Hemotherapy Department, Hospital Universitario Puerta de Hierro Majadahonda, Madrid, Spain. ${ }^{3}$ Internal Medicine Department, Infectious diseases unit, Hospital Universitario Puerta de Hierro Majadahonda, Madrid, Spain. ${ }^{4}$ Clinical Pharmacology Department, Hospital Clínic Barcelona, Medical Statistics core facility - IDIBAPS, Barcelona, Spain. ${ }^{5}$ Pneumology Department, Hospital Universitario Puerta de Hierro Majadahonda, Madrid, Spain. 'IIS Puerta de Hierro - Segovia de Arana, Madrid, Spain. ${ }^{7}$ Flu and Respiratory Virus Unit, Centro Nacional de Microbiología, Instituto de Salud Carlos III, Madrid, Spain. ${ }^{8}$ Hematology and Hemotherapy Department, Hospital Universitario Puerta de Hierro Majadahonda, Madrid, Spain.

Received: 31 July 2020 Accepted: 29 December 2020

Published online: 20 January 2021

\section{References}

1. Mair-Jenkins J, Saavedra-Campos M, Baillie JK, Cleary P, Khaw FM, Lim WS, et al. The effectiveness of convalescent plasma and hyperimmune immunoglobulin for the treatment of severe acute respiratory infections of viral etiology: a systematic review and exploratory meta-analysis. J Infect Dis. 2015;211(1):80-90

2. Arabi YM, Hajeer AH, Luke T, Raviprakash $\mathrm{K}$, Balkhy H, Johani S, et al. Feasibility of using convalescent plasma immunotherapy for MERS-CoV infection, Saudi Arabia. Emerg Infect Dis. 2016;22(9):1554-61.

3. DesJardin JA, Snydman DR. Antiviral immunotherapy: a review of current status. BioDrugs. 1998;9(6):487-507. https://doi.org/10.2165/00063030199809060-00006

4. Casadevall A, Pirofski LA. The convalescent sera option for containing COVID-19. J Clin Invest. 2020;130(4):1545-8.

5. Cheng Y, Wong R, Soo YOY, Wong WS, Lee CK, Ng MHL, et al. Use of convalescent plasma therapy in SARS patients in Hong Kong. Eur J Clin Microbiol Infect Dis. 2005;24(1):44-6.

6. Fu Y, Cheng Y, Wu Y. Understanding SARS-CoV-2-mediated inflammatory responses: from mechanisms to potential therapeutic tools. Virol Sin. 2020; 35(3):266-71. https://doi.org/10.1007/s12250-020-00207-4. Accessed 15 July 2020

7. Casadevall A, Joyner MJ, Pirofski LA. A randomized trial of convalescent plasma for COVID-19-potentially hopeful signals [published online ahead of print, 2020 Jun 3]. JAMA. 2020; https://doi.org/10.1001/jama.2020.10218.

8. ICH Topic E9 statistical principles for clinical trials. European Medicines Agency. [Internet]. 1998. http://www.ema.europa.eu/en/documents/ scientific-guideline/ich-e-9-statistical-principles-clinical-trials-step-5_en.pdf. Accessed 16 July 2020

9. Verbeke G, Molenberghs G. Linear mixed models for longitudinal data, springer series in statistics. 1st ed. New York: Springer-Verlag; 2000.

10. Brown H, Prescott R. Applied mixed models in medicine. 2nd ed. New York: John Wiley \& Sons, Ltd; 2006

11. Molenberghs G, Kenward MG. Missing data in clinical studies. Chichester, UK: John Wiley \& Sons, Ltd; 2007.

12. An EU programme of COVID-19 convalescent plasma collection and transfusion. European Comission. [Internet] 2020. https://ec.europa.eu/ health/sites/health/files/blood_tissues_organs/docs/guidance_plasma_ covid19_en.pdf. Accessed 12 February 2020.

13. Tetro JA. Is COVID-19 receiving ADE from other coronaviruses? Microbes Infect. 2020;22(2):72-3. http://www.ncbi.nlm.nih.gov/pubmed/32092539. Accessed 12 February 2020.

\section{Publisher's Note}

Springer Nature remains neutral with regard to jurisdictional claims in published maps and institutional affiliations.

\section{Consent for publication}

Not applicable.

\section{Competing interests}

The authors declare that they have no competing interests. 